\title{
c-Fos-MMP-9 pathway in central amygdala mediates approach motivation but not re- ward consumption
}

T. Lebitko ${ }^{1,2}$, J. Dzik $^{3}$, J. Jędrzejewska-Szmek ${ }^{3}$, M. Chaturvedi $^{2}$, T. Jaworski ${ }^{4}$, T. Nikolaev ${ }^{1}$, K. Meyza ${ }^{1}$, L. Kaczmarek², E. Knapska ${ }^{1, *}$

\section{Affiliations:}

${ }^{1}$ Laboratory of Emotions Neurobiology, BRAINCITY - Centre of Excellence for Neural Plasticity and Brain Disorders, Nencki Institute of Experimental Biology, Polish Academy of Sciences, Warsaw, Poland.

${ }^{2}$ Laboratory of Neurobiology, BRAINCITY - Centre of Excellence for Neural Plasticity and Brain Disorders, Nencki Institute of Experimental Biology, Polish Academy of Sciences Warsaw, Poland.

${ }^{3}$ Laboratory of Neuroinformatics, Nencki Institute of Experimental Biology, Polish Academy of Sciences Warsaw, Poland.

${ }^{4}$ Laboratory of Animals Models, Nencki Institute of Experimental Biology, Polish Academy of Sciences Warsaw, Poland.

*Correspondence to: e.knapska@nencki.edu.pl 


\begin{abstract}
:
Although impairments of motivational and consummatory aspects of reward behavior are core symptoms of several psychiatric disorders, the underlying neural and molecular mechanisms remain poorly understood. Here we report that blocking either c-Fos or matrix metalloproteinase 9 in the central amygdala disrupts approach motivation and appetitive discrimination learning, but not reward consumption. Further, we show that manipulation of c-Fos-MMP-9 pathway does not affect aversive motivation and learning. These findings reveal molecular mechanism of motivational anhedonia.
\end{abstract}




\section{INTRODUCTION}

Anhedonia, a core symptom of Major Depressive Episode (1), has long been defined as reduced ability to experience pleasure. This long standing definition was recently reconceptualized to include different deficits in reward processing, i.e., impairments in the ability to pursue or to experience pleasure $(2,3)$. Both components of reward processing, seeking and pleasure, are important factors for goal-directed reward-based learning (4). While the 'motivational' and 'consummatory' anhedonia is readily distinguished at the behavioral level, our understanding of the underlying neural circuitry and molecular underpinnings is insufficient (5), which may hamper the development of novel therapeutic approaches.

One of brain structures implicated in reward processing is the central nucleus of amygdala (CeA). It orchestrates a wide range of behavioral responses. Recent studies show that $\mathrm{CeA}$ is functionally heterogeneous and comprised of precisely connected, genetically defined cells, which select and control specific behaviors gating CeA outputs (6). It has been recently shown that GABAergic serotonin receptor 2a-expressing CeA neurons modulate food consumption (7), prepronociceptin-expressing cells promote rewarding properties of palatable food (8), and that GABA transporter GAT-3 mediates the choice of alcohol over a natural reward (9). Specific molecular players and neuronal circuits underlying approach motivation remain, however, unknown.

Proteases have been shown to mediate experience-dependent plasticity (10). In particular, our previous results suggested involvement of an extracellularly operating matrix metalloproteinase-9 (MMP-9) in CeA in appetitively, but not aversively, motivated learning (11). c-Fos, as a component of AP-1 transcription factor regulates gene expression of MMP-9, an enzyme involved in synaptic remodeling (12), necessary for, among others, reward-related learning (13). In the current study, we used RNAi-based approach to locally block c-Fos expression via lentiviral vector (14) and nanoparticles releasing a specific inhibitor to block 
MMP-9 activity in CeA (11) in order to elucidate the role of c-Fos-MMP-9 pathway in CeA in different subcomponents of reward-driven and aversively motivated behaviors. To confirm MMP-9 specificity of the manipulation we used a lentiviral vector inducing local, CeAspecific, overexpression of MMP-9.

\section{RESULTS}

Local blocking of $c$-fos expression in the central amygdala impairs reward motivation and sweetened water consumption but leaves avoidance motivation and bitter water consumption intact

Mice with local blockade of $c$-fos expression in the CeA with lentivector carrying shRNA (14) and control animals (Fig 1a,b) were subjected to discrimination training in IntelliCages (11). This protocol allows for balancing appetitive and aversive conditions and measurement of different components of reward-driven as well as aversively motivated behaviors. During the training, in one of four corners of the IntelliCage, animals had to discriminate between bottles (placed on two sides of the corner), which delivered either sweetened or tap water (appetitive task) or, alternatively, bitter (quinine-adulterated) or tap water (aversive task, Fig. 1c). Approach/avoidance motivation was measured as a number of nosepokes performed to bottles with sweetened/quinine-adulterated water and tap water (Fig. 1d,g), and consumption was measured as a percentage of licks of the sucrose/quinine solution to all recorded licks (Fig. 1e,h). To assess spatial discrimination learning we measured percentage of visits beginning with a correct response (to the bottle with sweetened water in the appetitively motivated task) or an incorrect response (to the bottle with quinine-adulterated water in the aversively motivated task) made during subsequent days of the training (Fig. 1f,i). Blocking of $c$-fos expression in the CeA impaired approach motivation, reward consumption, and ap- 
petitively motivated discrimination learning, leaving aversively motivated discrimination learning, avoiding motivation and consumption of quinine-adulterated water intact.

A

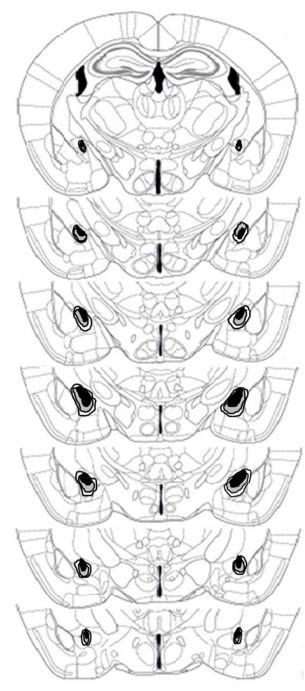

B
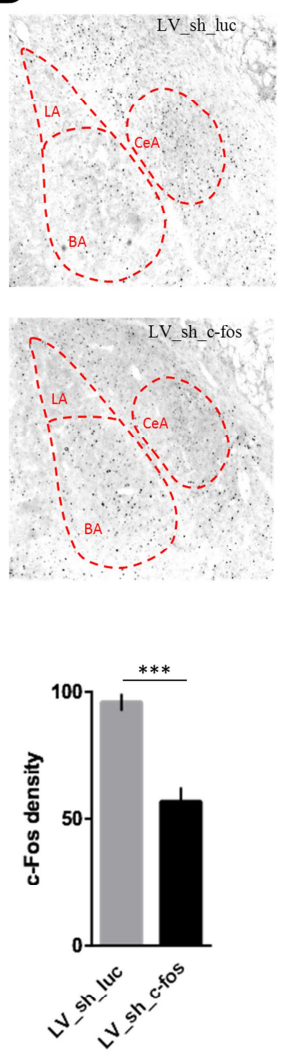

C

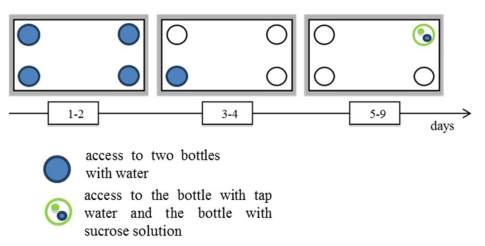

APPETITIVE

D

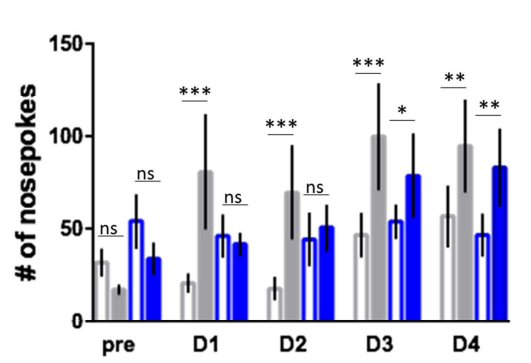

E

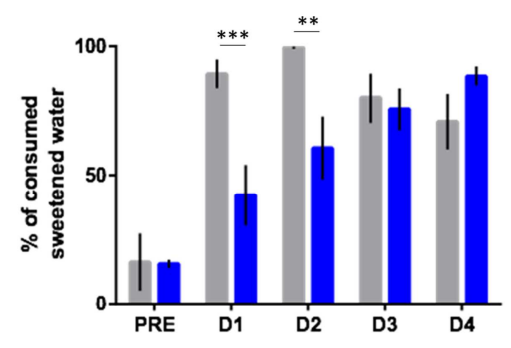

H

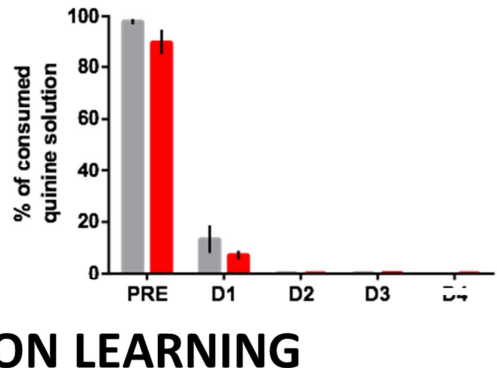

$\mathbf{F}$
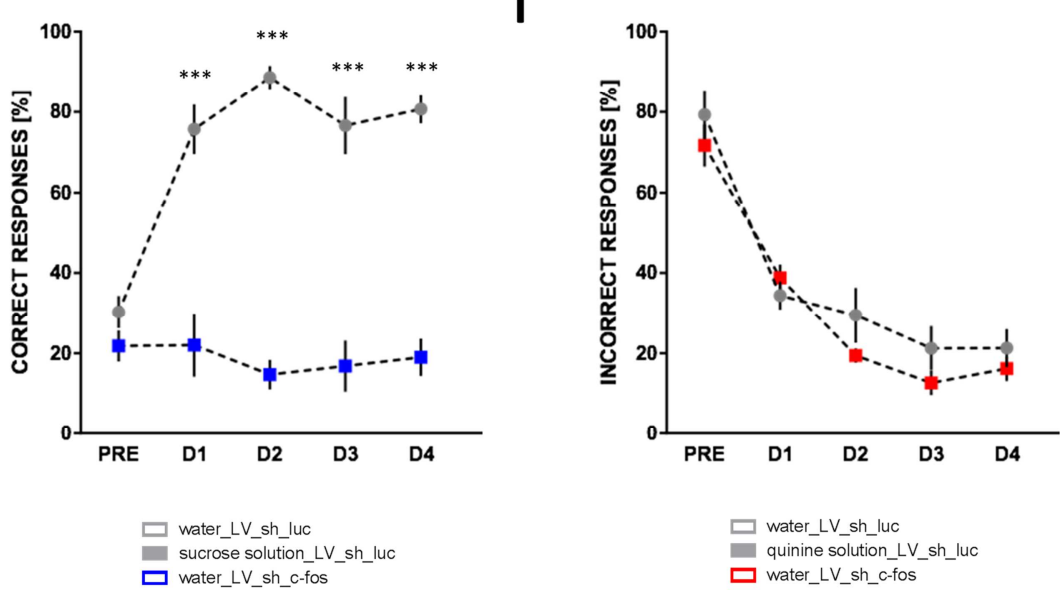

water_LV_sh_c-fos

sucrose solution_LV_sh_c-fos

\section{SEEKING}

G

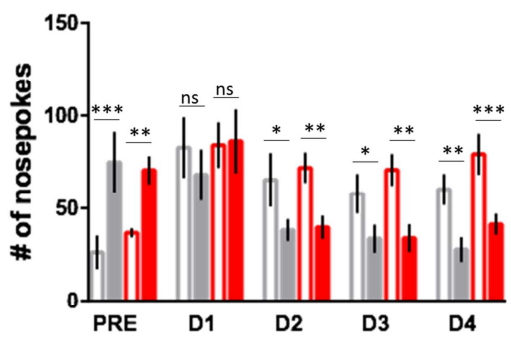

\section{CONSUMPTION}

access to two bottles
with water

access to the bottle with tap
water and the botle with
quinine solution

\section{AVERSIVE}

\section{DISCRIMINATION LEARNING}

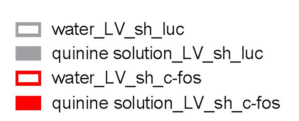


Fig. 1. A. Prior to the training mice were injected with $L V \_s h \_c-f o s(n=6)$ or LV_sh_luc $(\mathrm{n}=6)$ vectors to CeA. Maximal (white), minimal (black) and average (gray) transfection range within the injection sites for animals included in the analysis are shown. B. Efficacy of $c$-fos blocking. Immunohistochemical staining for c-Fos in amygdala and quantified density of c-Fos expression in the region transduced by LV-sh-luc and LV-sh-fos vectors $(\mathrm{F}(1,10)=$ 35.78, $\mathrm{p}=0.0001)$. C. Scheme of experiments in the IntelliCage. D. Reward seeking, measured as the number of nosepokes made to obtain sweetened water, was diminished in LV_sh_c-fos mice [group $\mathrm{x}$ day $\mathrm{x}$ solution: $\mathrm{F}(4,40)=3.15, \mathrm{p}=0.0244$ ]; statistical difference between number of nosepokes made to sweetened and tap water is shown (Duncan tests). E. LV_sh_c-fos mice consumed less sucrose solution than LV_sh_luc mice during first two days of the training [group: $\mathrm{F}(4,40)=6.20, \mathrm{p}=0.0006]$. F. Appetitively motivated discrimination learning was severely impaired in LV_sh_c-fos mice [group: F(1, 10)=96.60, p < 0.0001]. Avoidance motivation $(\mathbf{G})$, consumption of quinine-adulterated water $(\mathbf{H})$, and aversively motivated learning (I) were unaltered in LV_sh_c-fos mice. pre-adaptation period, D1-4, subsequent days of the training, CeA - central, LA - lateral, BA - basal nuclei of amygdala; bars denote SEM, * $\mathrm{p}<0.05, * * \mathrm{p}<0.01, * * * \mathrm{p}<0.001$

\section{Local blocking of matrix metalloproteinase 9 activity in the central amygdala impairs reward motivation but not sweetened water consumption, avoidance motivation and bitter water consumption}

Next, we measured approach and avoidance motivation, as well as sweetened and quinine-adulterated water consumption and learning in mice with MMP-9 activity locally inhibited in the CeA by tissue inhibitor of metalloproteinases 1 released from PLGA nanoparticles (TIMP-1, Fig. 2a,b) (11). Decreased activity of MMP-9 resulted in diminished approach motivation and impaired reward driven discrimination learning, leaving, however, consumption 
of sweetened water intact. Analogous to blocking of c-Fos expression, MMP-9 activity decrease did not affect the aversion towards and consumption of quinine-adulterated water, as well as aversively motivated discrimination learning (Fig. 2c-h). Similar motivational changes were observed in mice globally lacking $m m p-9$ activity, because of the unconditional gene knock-out (MMP-9 KO, Fig. S1).

\section{Local blocking of MMP-9 activity in CeA APPETITIVE \\ AVERSIVE}

A

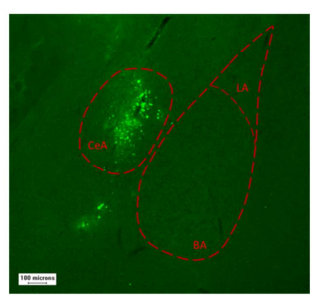

B

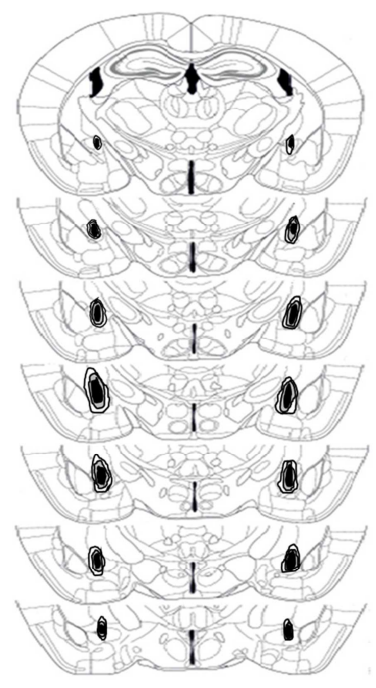

C

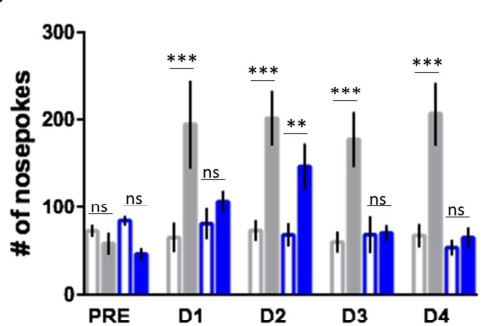

$F$

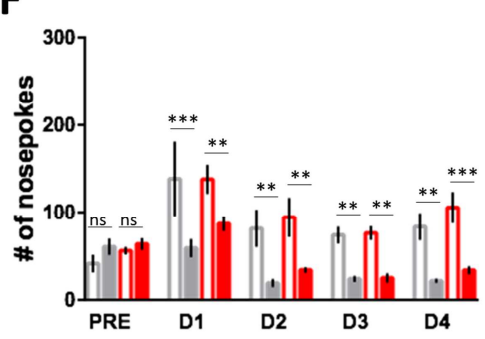

CONSUMPTION

D

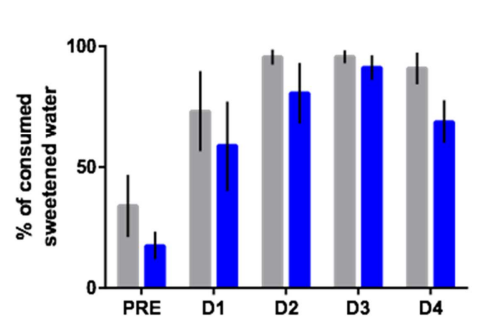

G

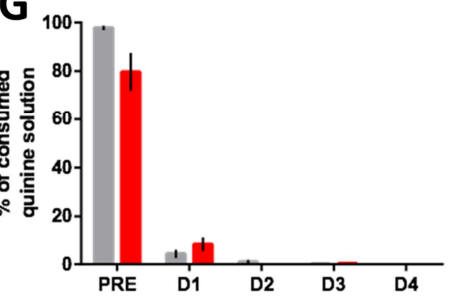

\section{DISCRIMINATION LEARNING}

$\mathbf{E}$
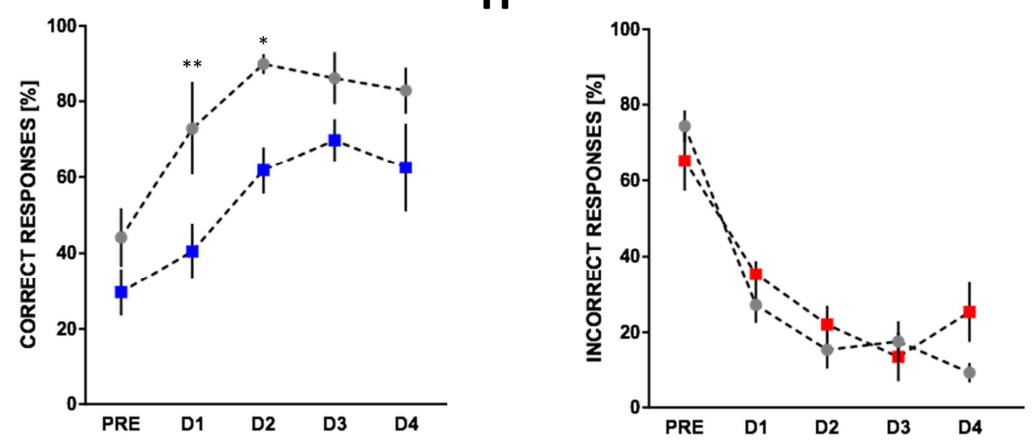

$\mathrm{H}$

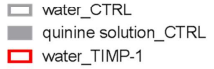
$\square$ water_TIMP-1 quinine solution_TIMP-1 
Fig. 2. To inhibit MMP-9 activity in CeA mice were injected with PLGA nanoparticles gradually releasing TIMP-1, an endogenous inhibitor of MMP-9 (n=6). Control animals (CTRL) were injected with nanoparticles carrying an inactive protein $(n=6)$. A. An example of an injection site and B. diffusion of nanoparticles within CeA are shown; maximal (white), minimal (black) and average (gray) injection sites for animals included in the analysis. C. Reward seeking was diminished in TIMP-1 mice [group: $\mathrm{F}(1,10)=12.62, \mathrm{p}=0.0052$ ]; statistical difference between number of nosepokes made to sweetened and tap water is shown (Duncan tests). D. There was no difference in consumption of sucrose solution between the TIMP-1 and CTRL groups. E. Appetitively motivated discrimination learning was impaired in TIMP1 mice [group: $\mathrm{F}(1,10)=25.37, \mathrm{p}=0.0005$ ]. Avoidance motivation $(\mathbf{F})$, consumption of quinine-adulterated water $(\mathbf{G})$, and aversively motivated learning $(\mathbf{H})$ were unaltered in TIMP-1 mice. pre-adaptation period, D1-4, subsequent days of the training, CeA - central, LA - lateral, BA - basal nuclei of amygdala; bars denote SEM, * $\mathrm{p}<0.05, * * \mathrm{p}<0.01, * * * \mathrm{p}<0.001$.

\section{Local overexpression of matrix metalloproteinase 9 in the central amygdala improves reward motivation but does not change sweetened water consumption, avoidance moti- vation and bitter water consumption}

Further, we tested whether an increase in MMP-9 level in the CeA affects motivation and learning. Lentiviral SYN-MMP9-GFP construct (15) was injected into the CeA, which resulted in a moderate overexpression of MMP-9 (Fig. 3a). The MMP-9 overexpression did not significantly change consumption of sweetened water and reward motivated discrimination learning. However, it increased approach motivation. Similarly to blocking of c-Fos expression and MMP-9 inhibition, MMP-9 level increase affected neither aversion towards nor consumption of quinine-adulterated solution, nor aversively motivated discrimination learning 
(Fig. 3c-h). Importantly, moderate increase in MMP-9 activity in the CeA led to motivational changes, but did not significantly affect reward-motivated learning, suggesting the primary role of motivational changes in the effects we observed when studying c-Fos-MMP-9 pathway.

\section{Local overpression of MMP-9 in CeA}

\section{APPETITIVE}

\section{AVERSIVE}

\section{SEEKING}

B

A
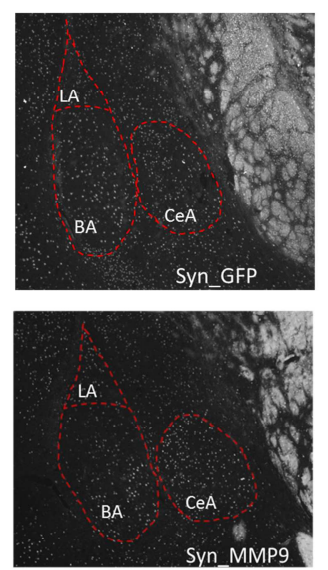

C
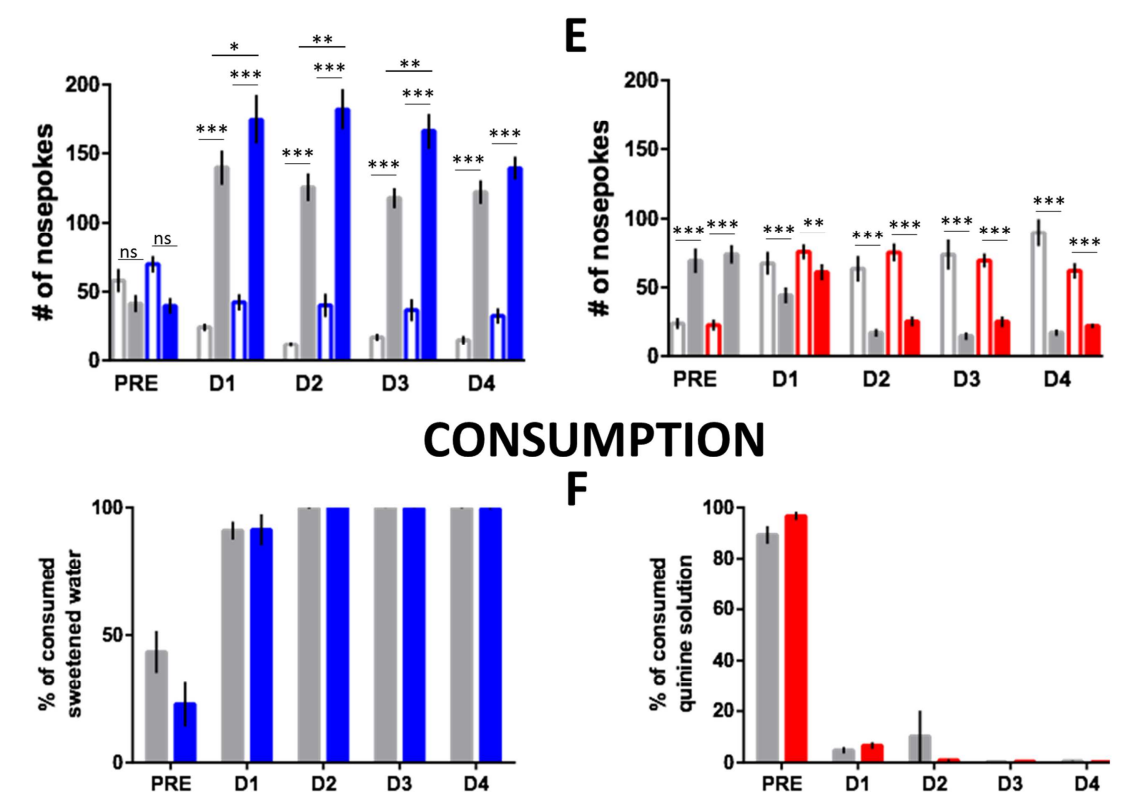

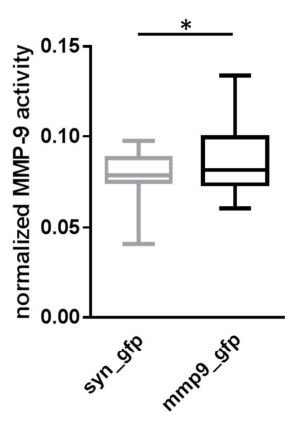

D

DISCRIMINATION LEARNING

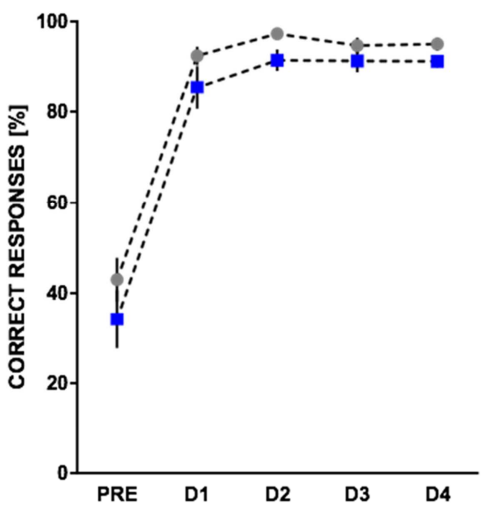

$\square$ water syn gfp

sucrose solution_syn_gfp 口 water_syn_mmp9_gfp

- sucrose solution_syn_mmp9_gfp
G

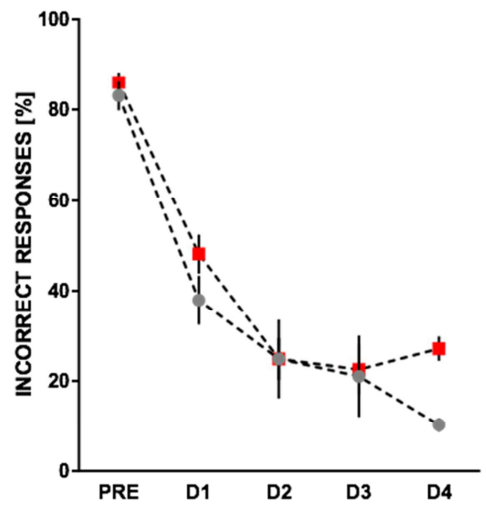

$\square$ water_syn_gfp

- quinine solution_syn_gfp water_syn_mmp9_gfp - quinine solution_syn_mmp9_gfp 
Fig. 3. To increase MMP-9 level in the CeA mice were injected with lentiviral vector carrying SYN-MMP9-GFP construct $(\mathrm{n}=10)$. The control animals were injected with SYN-GFP construct $(n=9)$. A. In situ zymography showed a moderate increase in MMP-9 expression in the CeA of SYN-MMP9-GFP mice (min to max graph, K-S test, $\mathrm{p}=0.0275$ ). B. Reward seeking was increased when MMP-9 was overexpressed in the CeA [group: $F(1,17)=7.38, p=0.0147$ ]; statistical difference between number of nosepokes made to sweetened and tap water is shown (Duncan tests). C. There was no difference in consumption of sucrose solution between the SYN-MMP9-GFP and SYN-GFP mice. D. Appetitively motivated discrimination learning was not changed in MMP-9 overexpressing group. Avoidance motivation (E), consumption of quinine-adulterated water $(\mathbf{F})$, and aversively motivated learning $(\mathbf{G})$ were unaltered in MMP9 overexpressing mice. pre-adaptation period, D1-4, subsequent days of the training, CeA central, LA - lateral, BA - basal nuclei of amygdala; bars denote SEM, * $\mathrm{p}<0.05,{ }^{*} * \mathrm{p}<0.01$, $* * * \mathrm{p}<0.001$

\section{DISCUSSION}

Here we report that motivation to pursue alimentary reward depends causally on cFos-MMP-9 pathway in the CeA. We show that blocking of c-Fos-MMP-9 pathway disrupts approach motivation, and that inhibition of MMP-9 alone does not impair reward consumption. We also show that modulation of c-Fos-MMP-9 pathway activity affects appetitive, but not aversive, motivation and learning. The results suggest that there are specialized molecular mechanisms associated with pursuing alimentary rewards in the $\mathrm{CeA}$, in line with the behavioral distinction between hedonic response to rewards and motivation to pursue them (3).

While discrimination learning is mediated by cortico-striatal systems (15), CeA has been implicated in modulation of incentive motivation to pursue a reward $(16,17)$. Our results confirm that link. Molecular pathways related to appetitive motivation have not been identi- 
fied before. Recently, Kim et al. (18) described distinct groups of c-Fos positive CeA neurons responding to stimuli of positive and negative valence. The function of $c$-fos expression and molecular mechanisms triggered by its activation in CeA neurons have, however, remained unclear. Our findings reveal a role of c-Fos-MMP-9 pathway in the modulation of approach motivation. Since c-Fos blocking caused more profound effects than MMP-9 inhibition or mmp-9 knock-out, including impairment of reward consumption, the results suggest existence of a different, MMP-9-independent, pathway in the CeA that regulates consummatory aspects of reward behavior. Motivational anhedonia, on the other hand, seems to be related to a malfunction of the MMP-9-dependent molecular mechanism.

Impaired motivation is a core feature of major depression, bipolar disorder, schizophrenia, as well as addiction (16-18). In particular, habitual drug seeking has been shown to depend on CeA (19). Since MMP-9 activity may be influenced pharmacologically (20), our results point to a potential target of intervention methods for motivational anhedonia.

\section{METHODS}

\section{Animals}

All procedures were carried out in accordance with the European Union and Polish guidelines for the care and use of laboratory animals, and were approved by the Local Ethical Committee. 7-8-week-old C57BL/6 mice were obtained from a commercial supplier and housed in a 12/12 h dark/light cycle, partially reversed (the dark phase shifted from 20:00-8:00 to 13:0001:00 or 12:00-24:00 dependent on daylight savings), with food available ad libitum.

\section{Lentiviral Vectors construction and preparation}

Vectors bearing shRNA coding sequences sh_luc and sh_cfos, and SYN-MMP9-GFP (SynaMMP9 T2A GFP) and SYN-GFP vectors were produced as previously described $(14,15)$. All vectors were prepared in the Laboratory of Animal Models in the Nencki Institute. 


\section{Formulation of poly(DL-lactide-co-glycolide) (PLGA) nanoparticles}

The PLGA nanoparticles (NPs) containing TIMP-1 (the endogenous MMP-9 inhibitor) or inactive protein (bovine serum albumin, BSA) were prepared as previously described (Chaturvedi et al., 2012). For fluorescent visualization the proteins were tagged with FITC (Sigma, F7250) before encapsulation.

\section{Stereotaxic injections}

Surgery room and surgical instruments were sterilized. Mice received subcutaneous Butomidor injection $(0.2 \mathrm{mg} / 100 \mathrm{~g})$, were placed on a heating pad and kept under isoflurane anesthesia until the end of the surgery. To keep eyes moist an ocular lubricant was used. The scalp was shaved, disinfected with $70 \%$ alcohol and incised to expose the skull. Two small holes were drilled to insert a Nanofil needle (NF36BV) into the CeA (anterior/posterior, $-0.12 \mathrm{~cm}$; medial/lateral, $\pm 0.26 \mathrm{~cm}$; dorsal/ventral, $-0.50 \mathrm{~cm}$, Paxinos and Franklin 2008). Lentiviral vectors $\left(4.5 \times 10^{7}\right.$ vector particles per site $)$ or nanoparticles $(0.5 \mu$ l total volume per site $)$ were bilaterally injected into CeA by pressure injection with a Nanofil syringe (10 $\mu \mathrm{l})$ attached to the infusion pump (MicroSyringe Pump, World Precision Instruments; $100 \mathrm{nl} / \mathrm{min}$ ). At the end of the injection the needle remained in place for additional 5 min for proper diffusion of the solution. All mice received Tolfedine $(0.08 \%, 0.1 \mathrm{ml}, 4.0 \mathrm{mg} / \mathrm{kg}$ subcutaneous $)$ and Enrofloxacin (Baytril $7.5 \mathrm{mg} / \mathrm{kg}$ ) for 2-4 days after surgery (depending on their condition) and were left undisturbed for two days (nanoparticles) or one month (lentiviral vectors) before the start of behavioral experiments.

\section{Behavioral apparatus}

All behavioral experiments were performed in the IntelliCage system, allowing for fully automated continuous testing of mice behavior (TSE Systems; https://www.tse-systems.com/). The apparatus consisted of a standard plastic cage $($ size $55 \times 37.5 \times 20.5 \mathrm{~cm}$ ), with four operant learning chambers, each fitting into every corner of the housing cage, and a sleeping shel- 
ter in the center. The feeder was located above the shelter (food ad libitum). Each chamber was equipped with an infrared motion/proximity detector and a transponder reader that allowed identification of individual mice. Only one mouse could use the operant chamber at a time. Each chamber was equipped with two openings, each permitting access to a drinking bottle. Both openings were blocked with automatically operated motorized door. Every event of a mouse poking its nose into the opening (a nosepoke response) and consumption of liquids (a lick response) were recorded for each mouse. Individual animals' access to the bottles was assigned depending on the schedule of the experiment (described below). The system was controlled by a computer and monitored from the experimenter's office via intranet. The mice were not disturbed, except for the technical breaks and exchange of bedding (once a week).

\section{Behavioral procedures}

The female mice were group-housed with a maximum of 12 animals per every IntelliCage. To identify individual mice in the IntelliCage, the animals were subcutaneously injected with a sterile, glass-covered microtransponders (11.5 mm length, $2.2 \mathrm{~mm}$ diameter; Trovan, ID-100) under a brief isoflurane anesthesia. The experiment started with an adaptation to the new environment of the cage (simple adaptation, 4 days). In that condition the doors were open, and the animals had free access to water in all corners. Then, the doors in all corners were closed and access to water was gained after performing a nosepoke (nosepoke adaptation, 4 days). Next, mice had access to bottles limited to only one corner of the IntelliCage (place preference, 2 days). At the start of the discrimination training, the bottle less preferred during the place preference period was replaced with the bottle containing sweetened water $(10 \%$ sucrose; appetitive discrimination training, 5 days). After the completion of the appetitive discrimination training mice had access to water in all four corners for 2 days. During the next 48 hours, mice had access to bottles only in one of the corners (which was different than the corner with the reward in the appetitive discrimination training). The bottle preferred during 
that phase was then replaced with a bottle containing quinine solution (0.005 M; bitter taste; aversive discrimination training, 5 days). Additionally, in the $c$-fos blocking experiment, after the completion of the aversive discrimination training the mice had gained access to tap water in all four corners (2 days). Next, the animals were water deprived for $22 \mathrm{~h}$ to increase their motivation to seek the reward. Then, in the corner least preferred during the preceding phase the mice had access to two bottles that contained sweetened water (10\% sucrose, on both sides of the corner). No access to water in other corners was offered. 90 minutes after completion of the training mice were sacrificed and their brains were taken for c-Fos expression analysis. The IntelliCage data were analyzed with PyMICE library written in Python programming language (RRID:nlx_158570; 21).

\section{Immunohistochemistry}

Mice were killed with an overdose of sodium pentobarbital $(100 \mathrm{mg} / \mathrm{kg})$ and perfused with ice-cold PBS (Merck) followed by 4\% PFA (Merck). The brains were collected, fixed overnight in $4 \%$ PFA at $4{ }^{\circ} \mathrm{C}$, and subsequently immersed in $30 \%$ sucrose at $4^{\circ} \mathrm{C}$. Then the tissue was frozen (isopentane, POCh on dry ice) and store at -80C until sectioning into $40 \mu \mathrm{m}$ sections on a cryostat. Coronal brain sections containing amygdaloid nuclei $(1.46-1.70 \mathrm{~mm}$ posterior to bregma, Paxinos and Franklin 2008) were collected. The immunohistochemical staining was performed on free-floating sections. The sections were first washed three times in PBS, incubated for $10 \mathrm{~min}$ in $0.003 \% \mathrm{H}_{2} \mathrm{O}_{2}$ in PBS, washed twice in PBS, and incubated with a polyclonal antibody (anti-c-Fos, 1:1000; Milipore, no. ABE457) and normal goat serum (3\%; Vector) in PBS for $48 \mathrm{~h}$ at $4^{\circ} \mathrm{C}$. Then, the sections were washed three times in PBST (PBS with $0.3 \%$ Triton X-100; Merck), incubated with goat anti-rabbit biotinylated secondary antibody (1:1000; Vector) and normal goat serum (3\%) in PBST for $2 \mathrm{~h}$ at RT. The sections were then washed three times in PBST, incubated with avidin-biotin complex (1:1000 in PBST; Vector ABC kit) for $1 \mathrm{~h}$ at RT. The immunostaining reaction was developed using the 
oxidase-diaminobenzidine-nickel method. The sections were washed three times in PBS and incubated in distilled water with diaminobenzidine (DAB; Merck), $0.5 \mathrm{M}$ nickel chloride for 5 min. The staining reaction was stopped by three washes with PBS. The reaction resulted in a brown-black stain within the c-Fos-positive nuclei of neurons. The sections were then mounted on slides, air dried, dehydrated in xylene, coverslipped with Entellan (Merck), and images containing CeA were collected with an optical microscope (Nikon Instruments). The c-Fos immunopositivity was measured as density, determined in the following manner. For each brain section, the number of c-Fos immunopositive nuclei in a given amygdalar structure was counted and divided by the area occupied by this structure (in $\mathrm{mm}^{2}$ ). Image analysis was done with the aid of an image analysis computer program (ImageJ) on at least two sections per animal.

\section{Histology}

Mice were killed by an overdose of sodium pentobarbital $(100 \mathrm{mg} / \mathrm{kg})$, perfused with ice-cold PBS (Merck) followed by 4\% PFA (Merck). The brains were collected, fixed overnight in 4\% PFA at $4^{\circ} \mathrm{C}$, and subsequently immersed in $30 \%$ sucrose at $4^{\circ} \mathrm{C}$. Then the brains were frozen and $40 \mu \mathrm{m}$ sections were cut on a cryostat. The free-floating brain sections were collected in PBS. For injection site verification, the sections were directly mounted onto glass slides with VECTASHIELD mounting medium (Vector Laboratories) with 4,6-diamidino-2phenylindole. A florescence microscope (Nikon Instruments) was used for imaging the brain sections.

\section{In situ zymography}

In situ zymography was done according to the protocol published earlier (Gawlak et al. 2009). In brief, coronal brain sections $(4 \mathrm{~mm})$ containing the amygdalar complex were dissected immediately after the animals have been sacrificed and placed in methanol:ethanol mix (3:7, at $\left.4^{\circ} \mathrm{C}\right)$ for $24 \mathrm{~h}$. Then they were transferred to: $90 \%$ ethanol $\left(1 \mathrm{~h}\right.$ at $\left.4^{\circ} \mathrm{C}\right), 100 \%$ ethanol $(2 \times 1 \mathrm{~h}$, 
$\left.4^{\circ} \mathrm{C}\right), 100 \%$ ethanol $\left(30 \mathrm{~min}, 37^{\circ} \mathrm{C}\right)$, polyester wax (VWR 360704E):ethanol $\left(1: 3,3 \mathrm{~h}, 37^{\circ} \mathrm{C}\right)$, polyester wax:ethanol $\left(1: 1, \mathrm{ON}+8 \mathrm{~h}, 37^{\circ} \mathrm{C}\right)$, polyester wax:ethanol $\left(9: 1, \mathrm{ON}, 37^{\circ} \mathrm{C}\right)$ and $100 \%$ polyester wax $\left(\mathrm{ON}, 42^{\circ} \mathrm{C}\right)$. Thereafter the tissue was placed at the bottom of a cylinder $(\varnothing$ $1 \mathrm{~cm})$ and embedded in fresh $100 \%$ polyester wax. When a wax column set $\left(30 \mathrm{~min}\right.$ in $\left.4^{\circ} \mathrm{C}\right)$ it was placed, tissue facing upwards in a molten paraffin (Sigma Aldrich 327204) block precooled to about $42-45^{\circ} \mathrm{C}$. When the paraffin block set $\left(30 \mathrm{~min}\right.$ in $\left.4^{\circ} \mathrm{C}\right)$ it was attached to a wooden frame and subsequently was stored at $-20^{\circ} \mathrm{C}$. Sectioning into $6 \mu \mathrm{m}$ coronal slices was done using a microtome equipped with the running water path (RMC, Boeckeler, Germany). Slides (Thermo Scientific Superfrost UltraPlus) with mounted brain slices were stored at $4^{\circ} \mathrm{C}$ until zymography in situ took place.

The in situ zymography reaction required de-waxing of the tissue $(2 \times 5 \mathrm{~min}+10 \mathrm{~min}$ in 100\%ethanol at RT), followed by marking around with PAP Pen (Abcam ab2601) and irrigation with $\mathrm{dH}_{2} \mathrm{O}$ (3 x fast changes $+3 \times 3$ min). Preincubation in $\mathrm{dH}_{2} \mathrm{O}(100 \mathrm{~min})$ took place in a wet chamber $\left(37^{\circ} \mathrm{C}\right)$. The reaction with DQ gelatine (diluted 1:100 in the buffer supplied by the manufacturer, Invitrogen/Molecular Probes EnzChek ${ }^{\mathrm{TM}}$ Gelatinase/Collagenase Assay Kit E12055, $45 \mathrm{~min})$ was also done in a wet chamber $\left(37^{\circ} \mathrm{C}\right)$. It was followed by a wash-out in PBS (3x5 min, RT, Sigma, pH=7.4). Upon drying the slices were sealed with Fluoromount $\mathrm{G}$ (Invitrogen 00-4958-02) and left ON to dry before imaging on Nikon (Ni-U) microscope.

\section{Statistics}

All results are presented as mean \pm s.e.m. and were analyzed by the GraphPad 6 (Prism) and Statistica 7.0 software. No statistical methods were used to predetermine sample sizes, but our sample sizes are similar to those reported in previous publications (10). Normality tests were performed before choosing the statistical test. Motivation was analyzed with three-way ANOVAs with repeated measures followed by post-hoc Duncan tests (Statistica 7.0). Consumption and discrimination learning were analyzed with two-way ANOVAs fol- 
lowed by post-hoc Fisher LSD tests (GraphPad 6). c-Fos expression in c-fos blocking experiment was analyzed with one-way ANOVA. $P<0.05$ was considered significant $(* P<0.05$, $* * P<0.01, * * * P<0.001)$. Injection sites, viral expression and nanoparticles presence were confirmed for all animals. Mice showing incorrect injection sites were excluded from the data analysis. Additionally, one mouse in $c$-fos blocking experiment was removed from the analysis due to the lack of nosepoking activity during the adaptation phase of the behavioral experiments and 2 mice were not included in aversively motivated discrimination learning analysis because the data were not collected due to technical problems. In the MMP-9 overexpression experiments one mouse was eliminated from the control group due to the lack of nosepoking and licking activity. 


\section{REFERENCES}

1. DSM-5, (available at https://www.psychiatry.org/psychiatrists/practice/dsm).

2. M. T. Treadway, D. H. Zald, Reconsidering anhedonia in depression: lessons from translational neuroscience. Neurosci Biobehav Rev. 35, 537-555 (2011).

3. K. C. Berridge, M. L. Kringelbach, Pleasure systems in the brain. Neuron. 86, 646-664 (2015).

4. A. Dickinson, B. Balleine, Motivational control of goal-directed action. Animal Learning \& Behavior. 22, 1-18 (1994).

5. M. DiLuca, J. Olesen, The cost of brain diseases: a burden or a challenge? Neuron. 82, 1205-1208 (2014).

6. J. P. Fadok, M. Markovic, P. Tovote, A. Lüthi, New perspectives on central amygdala function. Curr. Opin. Neurobiol. 49, 141-147 (2018).

7. A. M. Douglass, H. Kucukdereli, M. Ponserre, M. Markovic, J. Gründemann, C. Strobel, P. L. Alcala Morales, K.-K. Conzelmann, A. Lüthi, R. Klein, Central amygdala circuits modulate food consumption through a positive-valence mechanism. Nat. Neurosci. (2017), doi:10.1038/nn.4623.

8. J. A. Hardaway, L. R. Halladay, C. M. Mazzone, D. Pati, D. W. Bloodgood, M. Kim, J. Jensen, J. F. DiBerto, K. M. Boyt, A. Shiddapur, A. Erfani, O. J. Hon, S. Neira, C. M. Stanhope, J. A. Sugam, M. P. Saddoris, G. Tipton, Z. McElligott, T. C. Jhou, G. D. Stuber, M. R. Bruchas, C. M. Bulik, A. Holmes, T. L. Kash, Central Amygdala Prepronociceptin-Expressing Neurons Mediate Palatable Food Consumption and Reward. Neuron. 102, 1037-1052.e7 (2019).

9. E. Augier, E. Barbier, R. S. Dulman, V. Licheri, G. Augier, E. Domi, R. Barchiesi, S. Farris, D. Nätt, R. D. Mayfield, L. Adermark, M. Heilig, A molecular mechanism for choosing alcohol over an alternative reward. Science. 360, 1321-1326 (2018).

10. B. K. Attwood, J.-M. Bourgognon, S. Patel, M. Mucha, E. Schiavon, A. E. Skrzypiec, K. W. Young, S. Shiosaka, M. Korostynski, M. Piechota, R. Przewlocki, R. Pawlak, Neuropsin cleaves EphB2 in the amygdala to control anxiety. Nature. 473, 372-375 (2011).

11. E. Knapska, V. Lioudyno, A. Kiryk, M. Mikosz, T. Górkiewicz, P. Michaluk, M. Gawlak, M. Chaturvedi, G. Mochol, M. Balcerzyk, D. K. Wojcik, G. M. Wilczynski, L. Kaczmarek, Reward learning requires activity of matrix metalloproteinase-9 in the central amygdala. J. Neurosci. 33, 14591-14600 (2013).

12. G. W. Huntley, Synaptic circuit remodelling by matrix metalloproteinases in health and disease. Nat. Rev. Neurosci. 13, 743-757 (2012).

13. A. C. W. Smith, Y. M. Kupchik, M. D. Scofield, C. D. Gipson, A. Wiggins, C. A. Thomas, P. W. Kalivas, Synaptic plasticity mediating cocaine relapse requires matrix metalloproteinases. Nat. Neurosci. 17, 1655-1657 (2014). 
14. L. de Hoz, D. Gierej, V. Lioudyno, J. Jaworski, M. Blazejczyk, H. Cruces-Solís, A. Beroun, T. Lebitko, T. Nikolaev, E. Knapska, I. Nelken, L. Kaczmarek, Blocking c-Fos Expression Reveals the Role of Auditory Cortex Plasticity in Sound Frequency Discrimination Learning. Cereb. Cortex. 28, 1645-1655 (2018).

15. M. Stefaniuk, A. Beroun, T. Lebitko, O. Markina, S. Leski, K. Meyza, A. Grzywacz, J. Samochowiec, A. Samochowiec, K. Radwanska, L. Kaczmarek, Matrix Metalloproteinase-9 and Synaptic Plasticity in the Central Amygdala in Control of Alcohol-Seeking Behavior. Biological Psychiatry. 81, 907-917 (2017).

16. A. E. Whitton, M. T. Treadway, D. A. Pizzagalli, Reward processing dysfunction in major depression, bipolar disorder and schizophrenia. Curr Opin Psychiatry. 28, 7-12 (2015).

17. M. J. Owen, A. Sawa, P. B. Mortensen, Schizophrenia. The Lancet. 388, 86-97 (2016).

18. P. W. Kalivas, N. D. Volkow, The neural basis of addiction: a pathology of motivation and choice. Am J Psychiatry. 162, 1403-1413 (2005).

19. J. E. Murray, A. Belin-Rauscent, M. Simon, C. Giuliano, M. Benoit-Marand, B. J. Everitt, D. Belin, Basolateral and central amygdala differentially recruit and maintain dorsolateral striatum-dependent cocaine-seeking habits. Nat Commun. 6, 10088 (2015).

20. B. Vafadari, A. Salamian, L. Kaczmarek, MMP-9 in translation: from molecule to brain physiology, pathology, and therapy. J. Neurochem. 139 Suppl 2, 91-114 (2016).

21. PyMICE: A Python library for analysis of IntelliCage data Behavior Research Methods (2017) doi: 10.3758/s13428-017-0907-5. 


\section{Acknowledgments}

Funding: EK laboratory has been supported by European Research Council Starting Grant (H 415148), and LK by the TEAM projects of Polish Foundation for Science. T.J. was supported by a grant from the National Science Center (2012/05/D/NZ3/02085). K.M. and T.N. were supported by a grant from the National Science Center (2015/18/E/NZ4/00600). MC was supported by "Mobilność Plus” fellowship from Polish Ministry of Science and Higher Education grant number 1291/MOB/IV/2015/0. Authors contributions: Concept and design E.K., LK; data acquisition T.L., M.C., T.J., T.N., K.M.: analysis and interpretation of data T.L., J.D., J.J-S., T.N., K.M., E.K.; drafting and revising the article E.K., L.K., K.M. Competing interests: The authors declare no competing financial interests in relation to the work described. Data and materials availability: The datasets generated during and/or analyzed during the current study are available from the corresponding author on reasonable request. 


\section{Supplementary Materials:}

\section{Supplementary methods}

\section{Animals}

All procedures were carried out in accordance with the European Union and Polish guidelines for the care and use of laboratory animals, and were approved by the Local Ethical Committee. Female 3-4 month-old homozygous mmp-9 knock-out mice on a C57BL/6J background (MMP-9 KO) and their wild-type (MMP-9 WT) littermates were used. The strain colony was maintained in the Animal House of the Nencki Institute. The animals were housed in a 12/12 $\mathrm{h}$ dark/light cycle, partially reversed (the dark phase shifted from 20:00-8:00 to 13:00-01:00 or 12:00-24:00 dependent on daylight savings), with food available ad libitum. 


\section{Global blocking of MMP-9 activity in CeA}

APPETITIVE

\section{SEEKING}

A

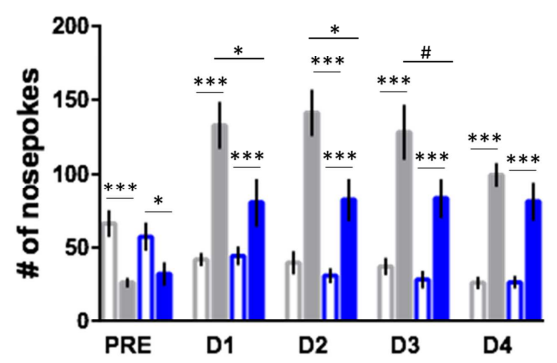

D

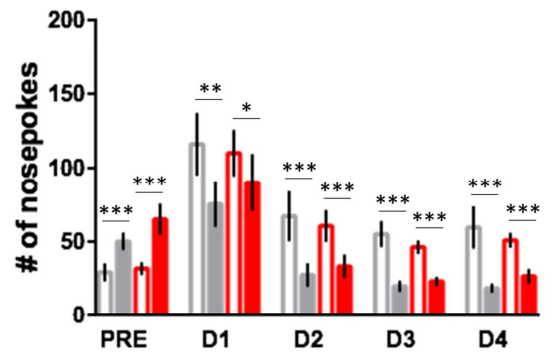

\section{CONSUMPTION}

B

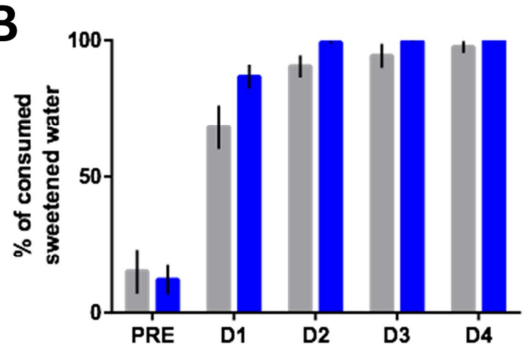

E

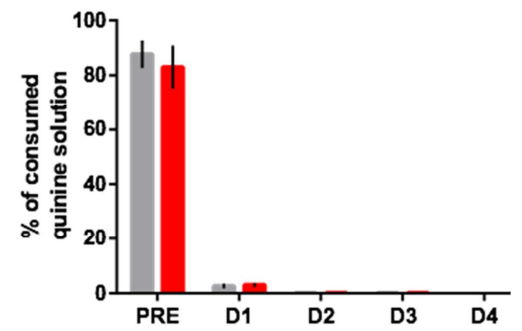

\section{DISCRIMINATION LEARNING}

C

$\mathbf{F}$
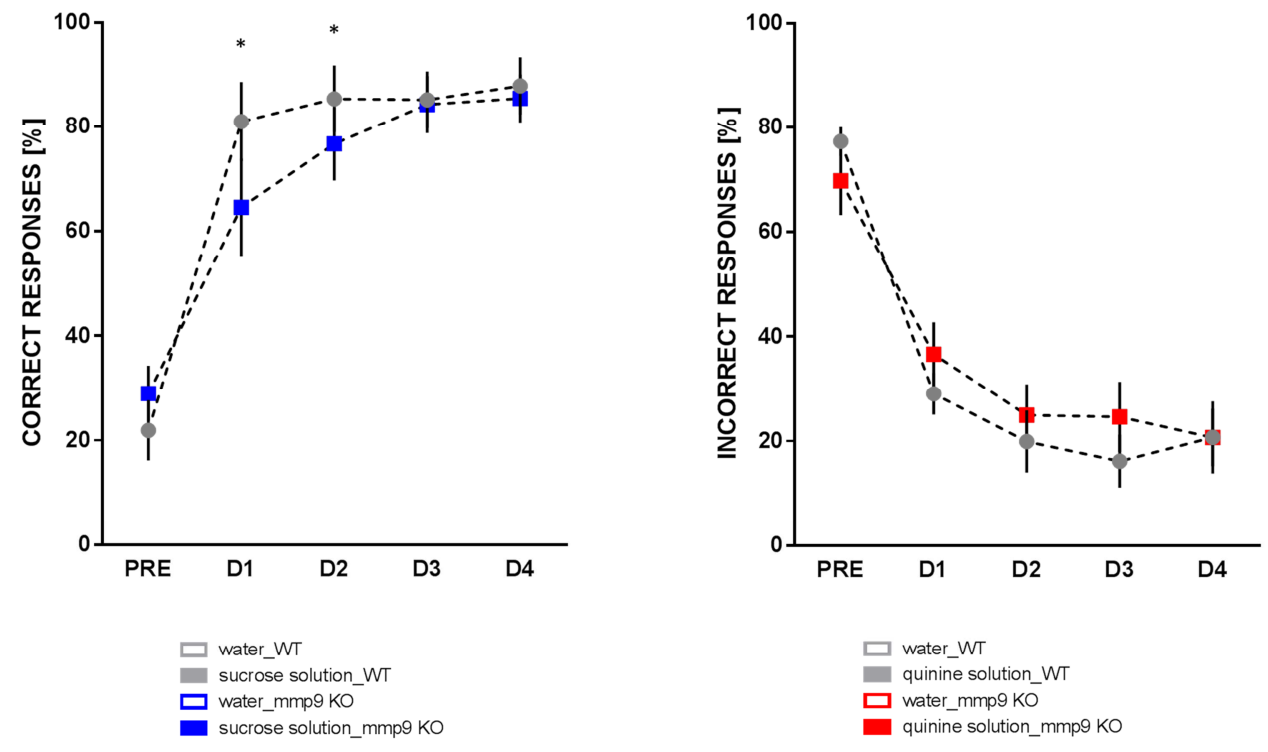

$\square$ water_WT

quinine solution_WT

$\square$ water_mmp9 KO

quinine solution_mmp9 KO

Fig. S1. To test the effects of global lack of MMP-9 activity we used the unconditional gene knock-out (MMP-9 KO n=12 and WT n=11). A. Reward seeking was diminished in MMP-9 
KO mice (group x session $x$ solution interaction: $F(4,84)=4,28, \mathrm{p}=0.0033$ ). B. There was no difference in consumption of sucrose solution between the genotypes. C. Appetitively motivated discrimination learning was delayed in MMP-9 KO group [group x session interaction: $\mathrm{F}(4,84)=2.49, \mathrm{p}=0.0490$; distribution normalized with arcsin transformation]. Avoidance motivation (D), consumption of quinine-adulterated water $(\mathbf{E})$, and aversively motivated learning (F) were unaltered in MMP-9 KO mice. pre-adaptation period, D1-4, subsequent days of the training; bars denote SEM, $* \mathrm{p}<0.05, * * \mathrm{p}<0.01, * * * \mathrm{p}<0.001$. 